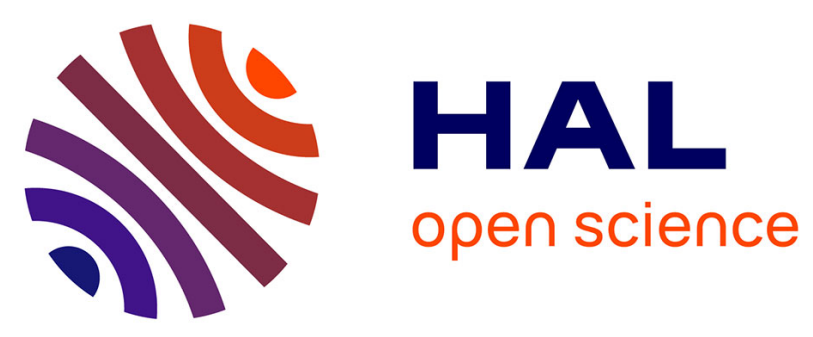

\title{
Association of impaired renal function with venous thrombosis: A genetic risk score approach
}

Romain Charmet, Astrid van Hylckama Vlieg, Marine Germain, Ronan Roussel, Michel Marre, Stéphanie Debette, Philippe Amouyel, Jean-François

Deleuze, Samy Hadjadj, Frits Rosendaal, et al.

\section{To cite this version:}

Romain Charmet, Astrid van Hylckama Vlieg, Marine Germain, Ronan Roussel, Michel Marre, et al.. Association of impaired renal function with venous thrombosis: A genetic risk score approach. Thrombosis Research, 2017, 158, pp.102 - 107. 10.1016/j.thromres.2017.08.015 . hal-01581823

\section{HAL Id: hal-01581823 https://hal.sorbonne-universite.fr/hal-01581823}

Submitted on 8 Sep 2017

HAL is a multi-disciplinary open access archive for the deposit and dissemination of scientific research documents, whether they are published or not. The documents may come from teaching and research institutions in France or abroad, or from public or private research centers.
L'archive ouverte pluridisciplinaire HAL, est destinée au dépôt et à la diffusion de documents scientifiques de niveau recherche, publiés ou non, émanant des établissements d'enseignement et de recherche français ou étrangers, des laboratoires publics ou privés. 


\section{Association of impaired renal function with venous thrombosis: a genetic risk score approach}

Romain Charmet ${ }^{1,2}$, Astrid van_Hylckama_Vlieg ${ }^{3}$, Marine Germain ${ }^{1,2}$, Ronan Roussel $^{4,5}$, Michel Marre ${ }^{4,5}$, Stéphanie Debette ${ }^{6,7}$, Philippe Amouyel ${ }^{8}$, Jean-François Deleuze ${ }^{9,10}$, Samy ${ }^{11,12,13}$ Hadjadj, Frits R Rosendaal ${ }^{3}$, Pierre-Emmanuel Morange ${ }^{\uparrow 4,15}$, David-Alexandre Trégouët ${ }^{1,2}$

${ }^{1}$ Sorbonne Universités, UPMC Univ. Paris 06, Institut National pour la Santé et la Recherche Médicale (INSERM), Unité Mixte de Recherche en Santé (UMR_S) 1166, Team Genomics \& Pathophysiology of Cardiovascular Diseases, Paris, France;

${ }^{2}$ ICAN Institute for Cardiometabolism and Nutrition, Paris, France

${ }^{3}$ Department of Clinical Epidemiology, Leiden University Medical Center, Leiden, The Netherlands;

${ }^{4}$ Assistance Publique Hôpitaux de Paris, Hôpital Bichat, DHU FIRE, Départment de Diabétologie, Endocrinologie et Nutrition, Paris, France.

${ }^{5}$ Université Paris Diderot, Sorbonne Paris Cité, UFR de Médecine, Paris, France.

${ }^{6}$ INSERM UMR_S 1219, Bordeaux Population Health Research Center, University of Bordeaux, France

${ }^{7}$ Department of Neurology, Bordeaux University Hospital, Bordeaux, France.

8. Univ. Lille, Inserm, CHU Lille, Institut Pasteur de Lille, U1167 - RID-AGE - Risk factors and molecular determinants of aging-related diseases, F-59000 Lille, France

${ }^{9}$ Centre National Génotypage, Institut de Génomique, CEA, 91057 Evry, France;

${ }_{11}^{10} \mathrm{CEPH}$, Fondation Jean Dausset, Paris, France

${ }_{11}^{11}$ Université de Poitiers, UFR de Médecine et Pharmacie, Poitiers, France;

12 INSERM, CIC 1402 \& U1082, Poitiers, France;

${ }^{13} \mathrm{CHU}$ de Poitiers, Service d'Endocrinologie \& CIC 1402, Poitiers, France;

${ }^{14}$ Laboratory of Haematology, La Timone Hospital, Marseille, France;

${ }^{15}$ INSERM UMR_S 1062, Nutrition Obesity and Risk of Thrombosis, Marseille, France; Aix-Marseille University

Corresponding author: Trégouët David-Alexandre, INSERM UMR_S 1166, 91 boulevard de l'Hopital 75013 Paris, France. + 331407796 86; david.tregouet@upmc.fr

\section{Abstract}

\section{Objective}

The association between impaired kidney function and venous thrombosis has been previously reported but supportive data are still sparse. We here wish to strengthen this association by investigating, by use of a genetic risk score approach, whether single nucleotide polymorphisms (SNPs) known to decrease the estimated glomerular filtration rate (eGFR), a surrogate marker for renal dysfunction, are associated with increased risk of venous thrombosis.

\section{Approach and Results}

Fifty-one polymorphisms selected from the literature to robustly associate with eGFR were first tested for association with venous thrombosis in a French case-control collection of 1,953 patients and 2,338 healthy individuals. This led to the identification of a genetic risk score based on 9 polymorphisms that strongly associated with increased risk (Odds Ratio 
$\left.(\mathrm{OR})=1.09[1.06-1.15], \mathrm{p}=1.4410^{-7}\right)$. This genetic score association replicated $(\mathrm{OR}=1.18$ [1.11-1.26], $p=8.8610^{-8}$ ) in an independent sample of 1,289 patients and 1,049 healthy controls part of the Dutch MEGA study. We then categorized the genetic score distribution observed in the combined samples into quintiles. Compared with the lowest quintile, the OR for increased risk of disease associated with the second, third, fourth and fifth quintiles were 1.13 [0.94 - 1.16], 1.47 [1.22 - 1.77], 1.52 [1.26 - 1.82] and 1.70 [1.41 - 2.05], respectively.

\section{Conclusions}

Using a genetic risk score analysis, our study provides new elements supporting the association between impaired renal function and the risk of venous thrombosis.

\section{Highlights}

- We identified a genetic risk score based on 9 eGFR-associated SNPs that impact on the risk of VT.

- Individuals with a score higher than 11 are associated with a 1.42 increased risk of disease

- Biological mechanisms underlying this association need to be further investigated

Keywords: genetics, estimated glomerular filtration rate, renal function, venous thrombosis, genetic risk score, association study

\section{Abbrevations :}

CKD: Chronic Kidney Disease

eGFR: estimated Glomerular Filtration Rate

GRS: Genetic Risk Score

GWAS: Genome Wide Association Study

SNP: Single Nucleotide Polymorphisms

VT: Venous Thrombosis

\section{Introduction}

Venous thrombosis (VT), that encompasses both deep vein thrombosis and pulmonary embolism, is the second most common cardiovascular disease and the third in terms of mortality. Its incidence is estimated to be 200 per 100000 person-years in Europe ${ }^{1}$ and its recurrence rate within 10 years is close to $30 \%$. The disease also represents a significant spending in health care ${ }^{2}$, it is for example associated with a total annual cost ranging from $€ 1.5$ to $13.2 €$ billion for the EU. ${ }^{3}$ The nature of the complex pathophysiological mechanisms that lead to VT is not fully characterized. Disturbances of the coagulation and fibrinolysis cascade leading to hypercoagulable states are clearly the most important contributor to VT etiology. Obesity-related mechanisms, inflammation ${ }^{4}$, platelets ${ }^{5}$ and complement cascade ${ }^{6}$ are additional biological systems that contribute to the development of the disease. However, despite these results of intensive research efforts, there are so far limited prognosis tools that help clinicians to predict which individuals are at risk for a VT event. More investigations are thus needed to better disentangle the etiological architecture underlying VT.

A meta-analysis of 5 community-based cohorts has shown that decreased estimated glomerular filtration rate (eGFR) was associated with increased VT risk ${ }^{7}$. It was further hypothesized that this association was partially mediated by elevated levels of coagulation Factor VIII and von Willebrand factor. ${ }^{8}$ Inflammation and coagulation biomarkers are associated with decreasing kidney function in ambulatory adults without established cardiovascular disease or chronic kidney disease. Investigations in the ARIC study ${ }^{9}$ demonstrated that increased levels of factor VIIlc, fibrinogen, and von Willebrand Factor were significantly associated with increased risk of incident chronic kidney disease, of which decreased eGFR is a surrogate marker, in European Americans. The pathophysiology 
underlying the association of hemostatic factors to kidney function decline is unclear, although triggers of hemostatic activation, including vascular injury, endothelial dysfunction, and inflammation, have been proposed as potential mechanisms.

To provide additional support to this hypothesis, we here propose the first genetic risk score approach addressing where genetic polymorphisms that have been robustly associated with eGFR, a marker for renal function, associate with VT risk.

Main outlines of the workflow are summarized in Figure 1. In a first step, we sought the literature for single nucleotide polymorphisms (SNPs) robustly associated with eGFR. These SNPs were then tested for association with VT risk in 1,953 VT patients and 2,338 healthy individuals from a French case control study (MARTHA/EOVT). ${ }^{10}$ We derived a genetic risk score (GRS) based on the VT-associated SNPs and tested it for replication in an independent sample of 1,289 VT patients and 1,049 controls from a Dutch case control study (MEGA study). ${ }^{11}$ We finally confirmed the association of the derived GRS with eGFR in a third independent study.

\section{Material \& Methods}

\section{Studied populations}

Informed consent was obtained from all participants in accordance with the Declaration of Helsinki, and the study met all institutional ethics requirements.

\section{Discovery VT case-control samples}

The discovery cohort was composed of 1,953 VT patients and 2,338 healthy individuals from two French VTE case-control studies, MARTHA and EOVT that have been extensively described before. ${ }^{10,12}$ Patients were individuals with documented personal VTE history and lacking strong genetic risk factors (Antithrombin, Protein $\mathrm{C}$ or Protein $\mathrm{S}$ deficiencies, FV Leiden homozygosity, FII G20210A homozygosity). Controls were apparently healthy individuals free of any chronic conditions and of a personal VT history.

Replication VT case-control samples

The replication stage was based on the same data from the Multiple Environmental and Genetic Assessment of risk factor for venous thrombosis (MEGA) study ${ }^{11}$ as those that were used in a recent meta-analysis of Genome-Wide Association Study (GWAS) data for VT ${ }^{12}$. 1,289 Dutch VT patients with no prior event of VT and no cancer were compared with 1,049 controls genetically matched for geographical ancestry. ${ }^{12}$

Additional validation cohorts

The Genesis/Genediab study group was composed of 1,370 individuals with Type 1 diabetes from the French population. ${ }^{13}$

Clinical characteristics of this population are provided in Supplementary Table A.

\section{Genotyping}

All individuals used in the work have previously been typed for genome-wide genotype SNPs using dedicated DNA microarrays and imputed with 1000 Genomes reference. Genotyping, quality controls and imputation analyses of the genotype data have been previously described in ${ }^{12}$ for the MARTHA, EOVT and MEGA studies and in ${ }^{14}$ for Genesis/Genediab.

\section{SNP selection}

Sixty-nine SNPs were identified to robustly associate with eGFR in different GWAS studies.

${ }^{15-18}$ These were selected as they have demonstrated genome-wide significance $\left(p<510^{-8}\right)$ association with eGFR and replicated in independent studies. From this list of candidate SNPs, we discarded SNPs from any pair of SNPs in linkage disequilibrium and excluded SNPs with bad imputation quality $\left(r^{2}<0.3\right)$ in both French GWAS cohorts. This resulted in 51 candidate SNPs (Supplementary Table B). 


\section{Statistical methods}

Derivation of the Genetic Risk Score (GRS)

Each of the 51 selected candidate SNPs was tested for association with VT in the French case-control samples using logistic regression analysis where the expected number of alleles (often referred to as imputed dose) at each SNP was used as a covariate in a model adjusting for age, sex and principal components derived from genotype data. Of note all SNPs had imputation quality $r^{2}>0.30$. From these analyses, we selected only SNPs for which the risk-allele was the one that was reported in the literature to associate with eGFR decrease, as compatible with the hypothesized relationship between renal dysfunction and VT risk. Only SNPs (Supplementary Table B) satisfying this condition were kept for the subsequent GRS analysis. In order to identify the most parsimonious and information SNP combination with respect to VT, an Akaike Information Criterion (AIC) ${ }^{19}$ strategy was adopted using a backward logistic regression procedure, while adjusting for sex, principal components and French sub-study group. SNPs selected by this AIC procedure were then entered into a GRS defined, for each individual, as the sum of the imputed risk-allele dose at each selected SNP. We confirmed that selected SNPs were also well imputed in the replication MEGA study.

Association of the GRS with VT risk

Association of the derived GRS with VT was then tested using logistic regression analysis using the same covariates as for the AIC strategy, both in the discovery (MARTHA/EOVT) and replication (MEGA) studies. Results observed in the discovery and replication studies were then pooled using fixed effect model (as implemented in the rmeta R package).

Association analysis with eGFR

The association of the proposed GRS was investigated in relation to eGFR in the Genesis/Genediab study where eGFR was estimated using plasma creatinin and the CKDEPI equation. ${ }^{20}$ Association was tested using linear regression analyses adjusted for age, sex and principal components derived from genotype data.

\section{Results}

From the 51 independent SNPs reported to robustly associate with eGFR in GWAS studies, only 27 presented with an eGFR decreasing allele that was also associated with an increased risk of VT (Supplementary Table B). The AIC based strategy applied to these 27 SNPs further selected a subset of 9 SNPs (Table 1) as the most parsimonious and informative group of SNPs to discriminate between patients and controls in the French populations. The minimal p-value of association of these 9 SNPs with VT in the French study was $p=0.011$ for the $D D X 1$ rs807601 and the highest $p$-value was $p=0.255$ for the SLC34A1 rs6420094 (Table 1).

A GRS variable was then derived from these 9 SNPs for each individual as the expected number of alleles associated with decreased eGFR levels carried by this individual. The distribution of the GRS in the French populations was shown in Supplementary Figure 1A. The GRS was significantly higher in VT patients than in controls $(10.4 \pm 0.04$ vs $10.1 \pm 0.047$, $\left.p=1.0310^{-7}\right)$. One unit increase of the GRS was associated with an increased risk for VT of $1.09[1.06-1.15]\left(p=1.4410^{-7}\right)$. In an independent collection of 1,289 Dutch patients and 1,049 controls, the proposed GRS exhibited a distribution similar to that observed in the French population (Supplementary Figure 1B). One unit increase of the GRS was found associated with an increase in VT risk of $1.18[1.11-1.26]\left(p=8.8610^{-8}\right)$. The GRS association did not differ between the two studies $(p=0.06)$ and the combined Odds Ratio (OR) for VT associated with one unit increase of the GRS was $1.12[1.09-1.16](p=3.7710$ $\left.{ }^{13}\right)$. When individual data from French and Dutch populations were combined together, the corresponding OR was 1.10 [1.06-1.14].

Figure 2 displayed the association of the GRS with VTE according to the quintiles of its distribution. In the French samples, the association was not completely linear as individuals in the $3^{\text {rd }}, 4^{\text {th }}$ and $5^{\text {th }}$ GRS quintiles were at similar risk of VT, OR $=1.39$ [1.12 - 1.73], 1.32 
[1.06 -1.64] and 1.54 [1.24 - 1.92] respectively, compared to individuals in the lowest quintile. This association was not completely linear in the Dutch population either. When the GRS distribution observed in the Dutch population was divided according to the same GRS quintiles as those used in the French studies, individuals in the $4^{\text {th }}$ and $5^{\text {th }}$ highest quintiles were at increased odds of 2.20 [1.54 - 3.14] and 2.23 [1.56 - 3.19], respectively, compared with individuals in the lowest quintiles.

In the combined population, compared with lowest quintile, the OR for increased risk of VT associated with the second, third, fourth and fifth quintiles were 1.13 [0.94 - 1.36], 1.47 [1.22 - 1.77]), 1.52 [1.26 - 1.82] and 1.70 [1.41 - .05], respectively. Based on these observations and the GRS quintiles distribution, these results can be translated into a 1.42 [1.25 - 1.61] increased risk of VTE associated with a GRS greater than 11 in the combined studied populations. Corresponding ORs were 1.31 [1.13 - 1.51] and 1.78 [1.39 - 2.27] in the French and Dutch samples, respectively.

Note that one of the SNPs, rs6088580, included in the definition of the GRS, is in moderate linkage disequilibrium $\left(r^{2}=0.15 ; D^{\prime}=1\right)$ with the $r 8867186$ known to associate with VT risk. ${ }^{21}$ Nevertheless, the GRS still remained significantly associated with VT risk in the discovery $\left(\mathrm{OR}=1.10\right.$ for one unit increase, $\left.p=6.2410^{-7}\right)$ and replication case-control studies $(\mathrm{OR}=$ $1.17, p=1.2010^{-6}$ ) after adjustment for the rs867186. The resulting meta-analyzed OR was 1.12 [1.08 - 1.15]. In addition, when the rs6088580 was discarded from the GRS definition, the restricted GRS was still associated with VT in the French $(\mathrm{OR}=1.10$ for one unit increase, $p=7.0810^{-7}$ ) and the Dutch $\left(O R=1.18, p=5.4810^{-7}\right)$ populations. The metaanalyzed OR for VT associated with such restricted GRS was 1.13 [1.09 - 1.16].

SNPs defining the GRS had been previously reported to individually associate with eGFR but their joint effect have never been assessed. Therefore, we investigated the impact on eGFR levels of the proposed GRS in a collection of 1,325 patients with Type 1 diabetes. In this study, the distribution of the GRS was similar to those observed in the discovery and replication VT studies (Supplementary Figure 1C). In this study, only one of the 9 assessed SNPs, the STC1 rs3758086, showed nominal association with eGFR $(p=0.035)$, all other SNPs having association $p$-value $>0.05$ (Supplementary Table $C$ ). Nevertheless, one unit increase of the GRS was associated with a decrease of $-1.19[-1.92--0.39]\left(p=3.5410^{-3}\right)$ in eGFR levels (Supplementary Table C).

\section{Discussion}

Employing a two-phase discovery-validation strategy on individual genetic data of two independent studies, we identified a GRS derived from 9 SNPs that, collectively, strongly associated with the risk of VT. These 9 SNPs combined altogether define a GRS that associates with the risk of the disease, individuals with an elevated GRS greater than 11 being at 1.42 increased risk of VT, whereas individually these SNPs showed very modest associations with the disease. These SNPs map to loci (DDX1, CPS1, SKIL, SLC34A1, ZNF204P, STC1, FBXL20, SLC7A9, TP53INP2) that have not been clearly associated with VT in recent large scale association studies ${ }^{12,22}$ and whose associated biology is poorly documented with respect to VT. Nevertheless, it could be mentionned that genetic variations at the SLC34A1 locus have been found associated with Factor XII antigen levels. ${ }^{23}$ In addition, the CPS1 and STC1 genes both play role in the phosphate mechanisms of action and are involved in vascular angiogenesis. ${ }^{24,25,26}$ These observations could be of particular relevance and open novel research avenues in light of recent works emphasizing the emerging role of the polyphosphate / factor XII pathway in thrombosis ${ }^{27}$. Finally, rare mutations in SLC7A9 are known to cause cystinuria, which could link this gene to VT via some homocysteine associated mechanisms ${ }^{28}$. 
The SNPs defining this GRS had been individually associated with eGFR in previous large meta-analysis of GWAS studies and we here showed that, while these SNPs were mildly associated with eGFR in a moderate sample size study, the derived GRS was much more significantly associated with eGFR. However, the GRS impact on eGFR variability was rather modest , $R^{2}=0.67 \%$, a value that must be compared to the $3.22 \%$ of eGFR variability explained by the 53 SNPs found to significantly associate with eGFR in the recent GWAS studies. ${ }^{15}$ This observation indicates that the influence of the GRS on VTE risk could not be solely mediated by eGFR that is an imperfect surrogate marker of impaired renal function. The use of genetic score approach to evidence a causative association between impaired renal function and VT is appealing as the genetic risk score is fixed at birth for each individual and should thus be independent from confounders normally affecting eGFR such as age or body mass index. We did not observe any association between the proposed GRS and BMI, age or sex (data not shown).

However, several limitations need to be acknowledged. The main one relates to the fact that we do not have eGFR measurements in the studied case-control samples preventing us from conducting mediation analysis to assess whether the impact of the GRS on VT risk vanishes after adjusting for eGFR. Similarly, proteinuria or cardiac biomarkers were not available in our samples to assess how the proposed GRS associates with CKD. While we obtained strong evidence that the GRS is associated with VT risk and eGFR, we have so far no evidence of inflammatory, vascular or hemostatic markers that could be influenced by this GRS. We investigated its association with several biological traits available in the MARTHA participants (including D-dimers, endogenous thrombin generation, plasma antigen or activity levels of fibrinogen, coagulation factors II, VIII, and XI, von Willebrand factor, antithrombin, protein $\mathrm{C}$, protein $\mathrm{S}$, activated partial thromboplastin time, haemoglobin, and white blood cell and platelet counts) but without any robust findings (data not shown). Unfortunately, factor XII antigen had not been measured and, according to the point discussed above, its association with the proposed GRS deserves further investigations. It is important to stress that the GRS was derived from SNPs that have been found robustly associated with eGFR in several studies but without any evidence they could be functional. They likely serve as markers for the underlying functional variants that need to be identified. One could speculate that once these functional variants are identified, the GRS they define would likely be a more accurate marker of the risk of VT. All these points deserve further investigations as well as further validation of the proposed GRS in large prospective cohorts for incidence, but also for recurrence of the disease.

\section{Conclusion}

This work provided additional elements supporting the association of impaired renal function with increased risk of venous thrombosis. However, the mechanisms underlying this association risk are far to be understood and require further deep investigations.

\section{Acknowledgments}

We are grateful to Elise GAND (Pole DUNE - CHU Poitiers) for data-management, Ahmed Amine KASMI (INSERM CIC1402, Poitiers) for clinical management and CRB BB 0033-00068 for biological handling and shipment of DNA to genomic platform

\section{Sources of funding}

R.C was supported by a PhD grant from the Region lle de France (CORDDIM program). MARTHA genetics research program was supported by the GENMED Laboratory of Excellence on Medical Genomics (ANR-10-LABX-0013), the French Clinical Research Infrastructure Network on Venous Thrombo-Embolism (F-CRIN INNOVTE) and the ICAN Institute for Cardiometabolism and Nutrition (ANR-10-IAHU-05), three research programs managed by the National Research Agency (ANR) part of the French Investment for the 
Future initiative. The Juvenile Diabetes Research foundation (JDRF-I) funded the genotyping of the Genesis/Genediab population (Grant -Diabetic Nephropathy Initiative).

\section{Disclosures}

None

\section{References}

1. Heit JA, Spencer FA, White RH. The epidemiology of venous thromboembolism. $J$ Thromb Thrombolysis. 2016;41:3-14.

2. Grosse SD, Nelson RE, Nyarko KA, Richardson LC, Raskob GE. The economic burden of incident venous thromboembolism in the United States: A review of estimated attributable healthcare costs. Thromb Res. 2016;137:3-10.

3. Kröger K, Küpper-Nybelen J, Moerchel C, Moysidis T, Kienitz C, Schubert I. Prevalence and economic burden of pulmonary embolism in Germany. Vasc Med. 2012;17:303-9.

4. Lwaleed BA, Kazmi RS, Cooper AJ. Inflammation, Endothelial Dysfunction, and Thromboembolism. Semin Thromb Hemost. 2015;41:547-8.

5. Montoro-García S, Schindewolf M, Stanford S, Larsen OH, Thiele T. The Role of Platelets in Venous Thromboembolism. Semin Thromb Hemost. 2016;42:242-51.

6. Foley $\mathrm{JH}$. Examining coagulation-complement crosstalk: complement activation and thrombosis. Thromb Res. 2016;141:S50-4.

7. Mahmoodi BK, Gansevoort RT, Næss IA, Lutsey PL, Brækkan SK, Veeger NJ, Brodin EE, Meijer K, Sang Y, Matsushita K, Hallan SI, Hammerstrøm J, Cannegieter SC, Astor BC, Coresh J, Folsom AR, Hansen JB, Cushman M. Association of mild to moderate chronic kidney disease with venous thromboembolism: pooled analysis of five prospective general population cohorts. Circulation. 2012; 126(16):1964-71.

8. Ocak G, Vossen CY, Lijfering WM, Verduijn M, Dekker FW, Rosendaal FR, Cannegieter SC. Role of hemostatic factors on the risk of venous thrombosis in people with impaired kidney function. Circulation. 2014;129:683-91.

9. Bash LD, Erlinger TP, Coresh J, Marsh-Manzi J, Folsom AR, Astor BC. Inflammation, hemostasis, and the risk of kidney function decline in the Atherosclerosis Risk in Communities (ARIC) Study. Am J Kidney Dis. 2009;53:596-605.

10. Germain M, Saut N, Greliche N,et al. Genetics of venous thrombosis: insights from a new genome wide association study. PLoS One. 2011;6:e25581. 
11. Blom JW, Doggen CJ, Osanto S, Rosendaal FR. Malignancies, prothrombotic mutations, and the risk of venous thrombosis. JAMA. 2005;293:715-22.

12. Germain M, Chasman DI, de Haan H, et.al. Meta-analysis of 65,734 individuals identifies TSPAN15 and SLC44A2 as two susceptibility loci for venous thromboembolism. Am J Hum Genet. 2015;96:532-42.

13. Hadjadj S, Cariou B, Fumeron F, et.al. Death, end-stage renal disease and renal function decline in patients with diabetic nephropathy in French cohorts of type 1 and type 2 diabetes. Diabetologia. 2016 ;59:208-16.

14. Germain M, Pezzolesi MG, Sandholm N, et.al. SORBS1 gene, a new candidate for diabetic nephropathy: results from a multi-stage genome-wide association study in patients with type 1 diabetes. Diabetologia. 2015;58:543-8.

15. Pattaro C, Teumer A, Gorski M, et.al. Genetic associations at 53 loci highlight cell types and biological pathways relevant for kidney function. Nat Commun 2016;7:10023.

16. Okada Y, Sim X, Go MJ, et.al. Meta-analysis identifies multiple loci associated with kidney function-related traits in east Asian populations. Nat Genet 2012; 44:904-9.

17. Pattaro C, Köttgen A, Teumer A, et.al. Genome-wide association and functional follow-up reveals new loci for kidney function. PloS Genet. 2012;8:e1002584.

18. Köttgen A, Pattaro $C$, Böger CA, et.al. New loci associated with kidney function and chronic kidney disease. Nat Genet. 2010;42:376-84.

19. Akaike $\mathrm{H}$.. A new look at the statistical model identification. IEEE Transactions on Automatic Control 1974; 19: 716-723.

20. Levey AS, Stevens LA, Schmid CH, Zhang YL, Castro AF 3rd, Feldman HI, Kusek JW, Eggers P, Van Lente F, Greene T, Coresh J; CKD-EPI (Chronic Kidney Disease Epidemiology Collaboration). A new equation to estimate glomerular filtration rate. Ann Intern Med. 2009;150:604-12.

21. Dennis J, Johnson CY, Adediran AS, de Andrade M, Heit JA, Morange PE, Trégouët DA, Gagnon F. The endothelial protein C receptor (PROCR) Ser219Gly variant and risk of common thrombotic disorders: a HuGE review and meta-analysis of evidence from observational studies. Blood. 2012;119:2392-400.

22. Hinds DA, Buil A, Ziemek D, et.al. Genome-wide association analysis of self-reported events in 6135 individuals and 252827 controls identifies 8 loci associated with thrombosis. Hum Mol Genet. 2016;25:1867-74.

23. Williams FM, Carter AM, Hysi, et. al. Ischemic stroke is associated with the ABO locus: the EuroCLOT study. Ann Neurol. 2013;73:16-31.

24. Zlot C, Ingle G, Hongo J, et. al. Stanniocalcin 1 is an autocrine modulator of endothelial angiogenic responses to hepatocyte growth factor. J Biol Chem. 2003;278:47654-9.

25. Yoshiko Y, Aubin JE. Stanniocalcin 1 as a pleiotropic factor in mammals. Peptides. 2004;25:1663-9.

26. Summar ML, Gainer JV, Pretorius M, et.al. Relationship between carbamoylphosphate synthetase genotype and systemic vascular function. Hypertension. 2004;43:186-91. 
27. Nickel KF, Labberton L, Long AT, et.al. The polyphosphate/factor XII pathway in cancer-associated thrombosis: novel perspectives for safe anticoagulation in patients with malignancies. Thromb Res. 2016;141:Suppl 2:S4-7.

28. Ragone R. Homocystine solubility and vascular disease. FASEB J. 2002;16:401-4.

Figure 1 (One column.)

Main outlines of the adopted research strategy.

Figure 2 (Two columns.)

Association between quintiles of the eGFR based GRS and the risk of venous thrombosis in the French (left) and Dutch (right) populations. 
Table 1 Association with VT risk of the nine SNPs defining the identified GRS

\begin{tabular}{|c|c|c|c|c|c|c|c|c|c|c|}
\hline \multirow[b]{2}{*}{ SNP } & \multirow[b]{2}{*}{ Chromosome } & \multirow[b]{2}{*}{ Position } & \multirow[b]{2}{*}{ Locus } & \multirow{2}{*}{$\begin{array}{c}\text { eGFR } \\
\text { associated } \\
\text { allele }\end{array}$} & \multicolumn{3}{|c|}{ MARTHA + EOVT } & \multicolumn{3}{|c|}{ MEGA } \\
\hline & & & & & $\begin{array}{c}\text { Allele } \\
\text { frequency* }\end{array}$ & Odds ratio $\dagger$ & Pvalue & $\begin{array}{c}\text { Allele } \\
\text { frequency }\end{array}$ & Odds ratio & Pvalue \\
\hline rs807601 & 2 & 15793014 & DDX1 & $G$ & 0.65 & 1.14 & 0.011 & 0.66 & 1.17 & 0.288 \\
\hline rs1047891 & 2 & 211540507 & CPS1 & $A$ & 0.32 & 1.14 & 0.016 & 0.31 & 1.03 & 0.846 \\
\hline rs9682041 & 3 & 170091902 & SKIL & $\mathrm{T}$ & 0.86 & 1.15 & 0.051 & 0.86 & 0.96 & 0.821 \\
\hline rs6420094 & 5 & 176817636 & SLC34A1 & $G$ & 0.32 & 1.06 & 0.255 & 0.31 & 1.12 & 0.445 \\
\hline rs7759001 & 6 & 27341409 & ZNF204P & $A$ & 0.77 & 1.10 & 0.100 & 0.77 & 1.37 & 0.068 \\
\hline rs3758086 & 8 & 23714992 & STC1,ADAM28 & $A$ & 0.41 & 1.06 & 0.243 & 0.39 & 1.19 & 0.204 \\
\hline rs9916302 & 17 & 37499949 & FBXL20 & $\mathrm{T}$ & 0.70 & 1.09 & 0.116 & 0.71 & 1.15 & 0.373 \\
\hline rs12460876 & 19 & 33356891 & SLC7A9 & $\mathrm{T}$ & 0.57 & 1.09 & 0.084 & 0.57 & 1.05 & 0.699 \\
\hline rs6088580 & 20 & 33285053 & PIGU,TP53INP2 & $\mathrm{C}$ & 0.48 & 1.07 & 0.144 & 0.47 & 1.23 & 0.127 \\
\hline
\end{tabular}

* Allele frequency in the control population

†Odds ratio and $p$-value were adjusted for sex and principal components 


\section{Supplementary Figure 1}

Distribution of the genetic risk score in the three studied populations: A: MARTHA/EOVT; B: MEGA; C: Genesis/Genediab. In MARTHA/EOVT and MEGA studies, data on healthy

individuals are colored in black while grey boxes represent patients with venous thrombosis.

\section{Supplementary Table A}

Clinical characteristics of the Genesis/Genediab population

\section{Supplementary Table B}

Association analysis of 51 eGFR associated SNPs with the risk of venous thrombosis in two French case-control populations, MARTHA and EOVT

\section{Supplementary Table C}

Association of the eGFR based GRS and its individual SNP component with eGFR levels in the Genesis/Genediab study 
